\title{
Temperature Gradient Gel Electrophoresis as a Valuable Accessory Tool for Assessment of Dysbiosis in Crohn's Disease
}

\author{
Vanessa Rafaela de Carvalho', Rogeria Keller1,2, Ana Carolina da Silva Santos, \\ Josias Rodrigues ${ }^{1}$ \\ ${ }^{1}$ Laboratory of Medical Bacteriology, Department of Microbiology and Immunology, Institute of Biosciences of \\ the State University of São Paulo (UNESP), Botucatu, Brazil \\ ${ }^{2}$ University of Western São Paulo, Presidente Prudente, Brazil \\ Email: josias@ibb.unesp.br
}

Received 21 June 2016; accepted 17 July 2016; published 20 July 2016

Copyright (C) 2016 by authors and Scientific Research Publishing Inc.

This work is licensed under the Creative Commons Attribution International License (CC BY).

http://creativecommons.org/licenses/by/4.0/

(c) (i)

\begin{abstract}
Escherichia coli and other Proteobacteria are augmented and several other bacteria are diminished in Crohn's (CD) disease patients' intestine. This imbalance in bacterial species composition-termed dysbiosis-seems to be determinant of CD manifestation. Since a great part of intestinal bacteria are not cultivable, detection of CD dysbiosis is accomplished by molecular tools, involving sequences analysis of the 16SrRNA gene (16SrDNA) present in the patient's clinical samples, which can be done by sequencing or electrophoresis in denaturing gels of 16SrDNA amplicons. By analyzing, by temperature gradient gel electrophoresis (TGGE) and next generation sequencing of 16SV6-V8rDNA amplicons present in gram negative cultures from four distinct clinical samples of a control subject and a CD patient, this study demonstrates that both techniques were able to detect $E$. coli overgrowth and reduction in species richness in CD and that TGGE can discriminate sequences collectively labeled as "unclassified" in 16SrDNA databases. Although TGGE per se does not identify the sequences, the discriminatory power that it confers represents valuable accessory information to next generation DNA sequencing (NGS), and as such must be used as a NGS complementary tool.
\end{abstract}

\section{Keywords}

Dysbiosis, Proteobacteria, Escherichia coli, Crohn's Disease

\section{Introduction}

The human body is occupied by a diverse and abundant microbial community (microbiome), which although

How to cite this paper: de Carvalho, V.R., Keller, R., da Silva Santos, A.C. and Rodrigues, J. (2016) Temperature Gradient Gel Electrophoresis as a Valuable Accessory Tool for Assessment of Dysbiosis in Crohn's Disease. Advances in Microbiology, 6, 549-554. http://dx.doi.org/10.4236/aim.2016.68055 
might include potentially pathogenic organisms, exerts innumerous homeostasis favoring functions. Health depends on maintenance of microbial diversity and abundance, so that disequilibrium in microbial species composition can lead to some disorders, such as inflammatory bowel diseases (IBD) and Clostridium difficile-associated pseudomembranous colitis, for which reversion or attenuation of the bacterial imbalance by, for example, the administration of probiotics [1] or fecal transplantation [2] have been viewed as promising therapeutic strategies. Thus, effort has been dedicated to identify the microbes' species that inhabit the human body, their metabolic products, and the nature of the interaction that they keep with the host.

Over $90 \%$ of body's microbes are prokaryotes belonging to the Bacteria dominium, a great part of which is as yet unknown. Existence of many microbial species has for long been ignored. This was at least in part due to limitations in culturing them, that is, to accomplish their artificial reproduction in laboratory conditions. Methods for studying microorganism are divided in those which are dependent and those which are not dependent on laboratory cultures. Culture dependent methods detect only live organisms, whereas those which are independent of culture detect live or dead, cultivable or non-cultivable organisms. Culture independent methods consist basically of molecular and serological techniques which identify bacterial extracellular products, cell associated molecules, genes or whole genome.

Progress in the study of human microbiome has relied mainly on the analysis of 16SrRNA gene (16SrDNA) sequences, one of the culture independent molecular approaches. 16SrDNA is ca. 1.5-kb in size and encodes the corresponding rRNA molecule, which is involved in protein synthesis signaling [3] and is part of all prokaryote's small ribosome subunit. It consists of 9 variable alternating with conserved sequences [4] [5]. PCR using primers combining with conserved sequences amplifies one or more 16SrDNA variable regions yielding an amplicon of a given size which contains 16SrDNA of distinct bacteria taxa. The identification of each of a specific bacterial DNA within a 16SrDNA amplicon can be done by submitting the amplicon to electrophoresis in denaturing gradient gels, where they are identified by bands of characteristic mobility. Thereafter, the bands can be cut from the gel, purified and sequenced. Traditionally, sequencing of the bands has been done with the Sanger method, but with the development of next generation technologies (NGS), 16SrDNA bacterial identification has depended less or abolished the amplicon electrophoresis in denaturing gradient gels. This is because sequencing of $16 \mathrm{SrDNA}$ by NGS is more versatile, enables a larger number of samples analyses and the bacterial identification is faster, since the sequence information can be processed with tools available at databases, such as the Ribosomal Database Project [6] and Greengenes [7]. Nonetheless, since a great part of bacteria is still unknown, no information can be retrieved on the corresponding sequence from these database, other than "unassigned" or "unidentified" followed by a mention to a given taxa usually of high taxonomic level. For example, it is frequently not possible identify the number of sequences of lower taxonomic levels (family, order or genus) specified in a DNA amplicon sample as "unidentified Proteobacteria”. It is likely that by submitting this amplicon to denaturing gel electrophoresis, the corresponding unidentified sequences would be resolved in multiple bands, each corresponding to taxa of lower taxonomic levels. Therefore, denaturing gradient gel electrophoresis can give useful information for bacterial identification in the analysis of 16SrDNA amplicon and thus must be used at least as a complementary procedure to NGS. In this study, we evaluated the validity of temperature gradient gel electrophoresis (TGGE) of 16SrDNA amplicons for assessing gram negative bacterial communities in clinical samples from a Crohn's disease (CD) patient and a control subject.

\section{Materials and Methods}

\subsection{Bacterial Cultures}

Three biopsies and one stool culture from each subject were tested. These cultures were previously originated from the patient's gut biopsies or stools' inoculum in MacConkey broth and re-culturing of the growth in brain heart infusion broth, where they were stocked at $-80^{\circ} \mathrm{C}$ with $15 \%$ glycerol [8]. Cultures from Escherichia coli, Shigella sp., Hafnia sp., Pseudomonas sp., Proteus sp., Providencia sp., Morganella sp., Klebsiella sp., Enterobacter sp., and Campylobacter sp. were used as references to define a temperature gradient for a good bands resolution.

\subsection{DNA Purification and PCR}

DNA from both clinical samples and known cultures were purified with the QiAmp mini kit (Qiagen, Hilden, Germany) and used as a template for a PCR targeting the V6-V8 regions of the 16SrDNA, with U968 (GAACGCGAAGAACCTTAC) plus a 40 bases GC clamp and L1401 (GCGTGTGTACAAGACCC), as forward and re- 
verse primers, respectively. Amplification conditions as well as reaction components in the PCR mix are described elsewhere [9].

\subsection{TGGE}

TGGE of the 16SV6-V8rDNA amplicons was run in a $0.8 \mathrm{~mm}$ polyacrylamide $(6 \%$ [wt/vol] acrylamide/biscrylamide [37.5:1], $8 \mathrm{M}$ urea, 20\% [vol/vol] formamide, $2 \%$ [vol/vol] glycerol, $0.09 \%$ [vol/vol], TEMED and $0.4 \%$ [vol/vol] ammonium persulfate) gel in a TGGE mini system (Biometra, Göttingen, Germany) for $2 \mathrm{~h}$ at $130 \mathrm{~V}$, with $1 \times \mathrm{TAE}$ as the running buffer. Following electrophoresis, the gel was fixed, silver stained according to the TGGE mini system manufacturer's protocol, dried at room temperature and submitted to image capturing at the Gel doc EZ Imager (Biorad, Hercules, USA). Determination of the best gradient temperature for TGGE separation of the bands was carried out by submitting to electrophoresis, in a single well gel, a pool resulting from the mixture of individual reference bacterial cultures amplicons, under a steep $30^{\circ} \mathrm{C}-50^{\circ} \mathrm{C}$ gradient perpendicular to the running electrophoresis direction. This procedure enabled the identification of $40^{\circ} \mathrm{C}-44^{\circ} \mathrm{C}$ as the temperature interval where the amplicons were separated (Figure 1). After submitting these reference cultures amplicons to electrophoresis, where the gradient temperature was applied in parallel to the electrophoretic run, this gradient was confirmed as the parameter for the best resolution of the bands. Therefore, the above defined $40^{\circ} \mathrm{C}-44^{\circ} \mathrm{C}$ temperature interval was used as a gradient in a TGGE to compare bacterial diversity in 16SV6-V8rDNA amplicons from CD patient's and control's samples cultures. Gel manufacture, as well as voltage and time of the run were as described above.

\subsection{SrDNA Amplicons Sequencing}

Amplicons from the 16SV6rDNA gram negative cultures from two each of the control and 3 CD patient's biopsies were sequenced using the Ion Torrent NGS platform. The resulting sequences were then compared with previously deposited reads at the RDP data base [6], for taxa identification. PCR protocol and primers for the 16SV6rDNA gene amplification followed the work of Andersson et al. [5].

\section{Results}

\subsection{TGGE Band Profiles of Amplicons from the CD and Control Patient}

As can be seen on Figure 2, there was a clear difference in DNA bands profile from control and CD patient's

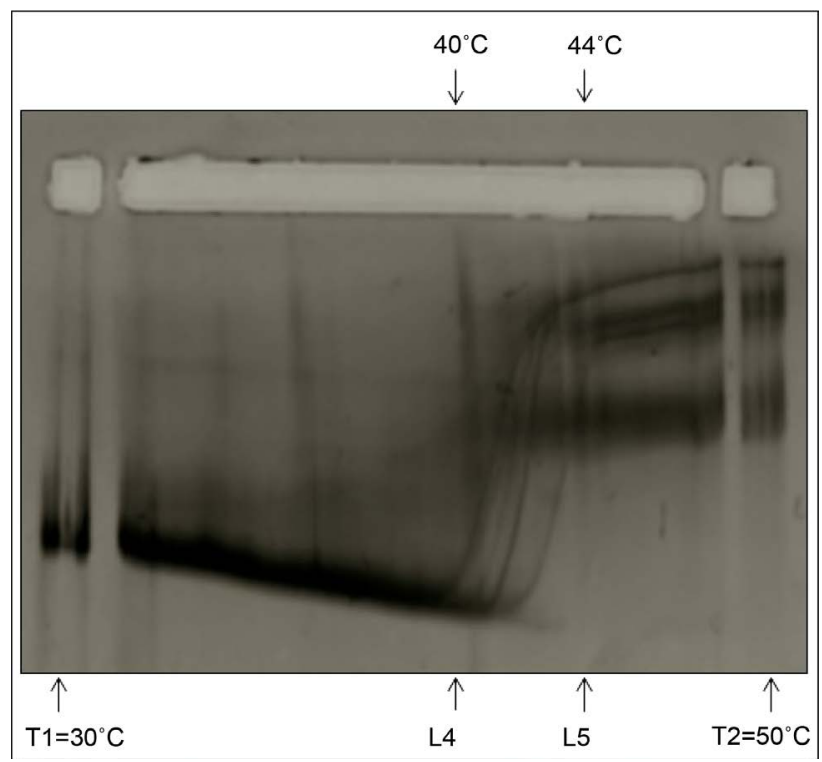

Figure 1. Perpendicular temperature gradient gel electrophoresis (TGGE) of reference gram negative bacterial cultures mixture (see text) of $16 \mathrm{SV6}$-V8rDNA amplicons in a $30^{\circ} \mathrm{C}-50^{\circ} \mathrm{C}$ temperature gradient, showing the gradient $\left(40^{\circ} \mathrm{C}-44^{\circ} \mathrm{C}\right)$ where the bands were separated. 


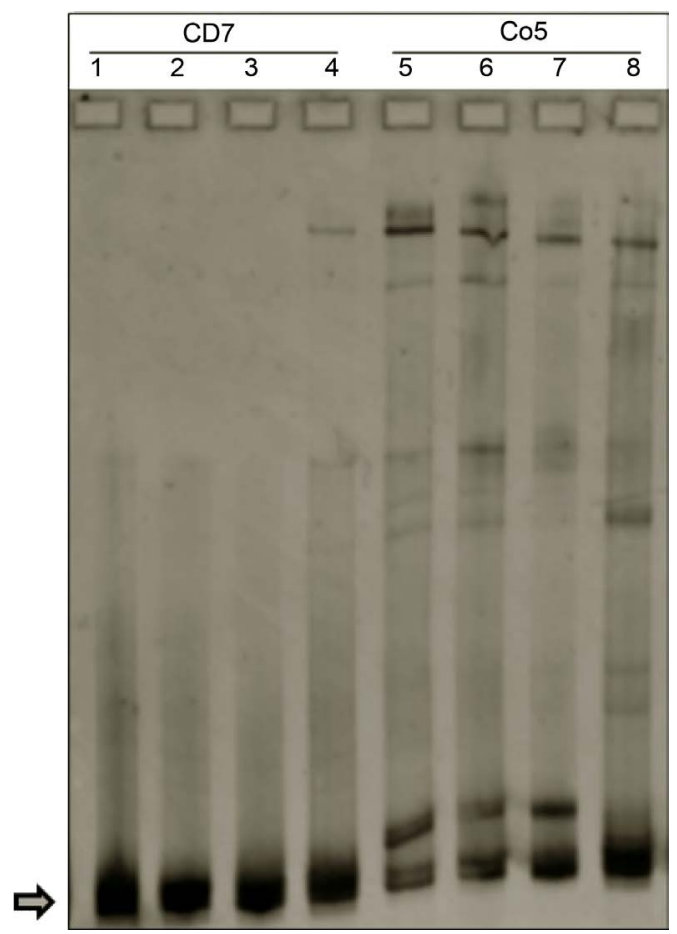

Figure 2. TGGE of 16SV6-V8rDNA amplicons of cultures from clinical materials originally grown on MacConkey broth of a Crohn's disease (CD7) patient and a control (Co5) subject. Arrow indicates bands with a electrophoretic mobility matching E. coli.

gram negative cultures, both in stools and biopsies samples. Except for stools, all other CD patient's samples yielded a single and intense band, with a mobility corresponding to E. coli, whereas all samples from control subject had multiple bands. Noticeably, in the control's samples, the band matching $E$. coli was much less intense, indicating the presence of a fewer amount of these bacteria's 16SV6-V8rDNA (Figure 2).

\subsection{Bacterial Identification}

As shown in Figure 3, the proportion of sequences assigned to Escherichia coli/Shigella in the control's cultures was inferior to that seen in cultures of CD patients' biopsies, revealing an enrichment of these bacteria in CD, relative to other Enterobacteriaceae. The sequenced 16SV6rDNA was from biopsies of the same control patient (Co5) of whom 16SV6-V8rDNA were analyzed by TGGE. The number of bacterial categories assigned to this patient in Figure 3 is inferior to the number of corresponding DNA bands in TGGE (Figure 2). Since the higher proportion of non E. coli reads in Figure 3 corresponds to unclassified Enterobacteriaceae, at least some of the bands in TGGE possibly represent taxa of this group which were indiscriminate by sequencing.

\section{Discussion}

The above results indicate that TGGE of 16SrDNA amplicons can give a good panoramic view of bacterial biodiversity in a clinical sample, enabling comparative analyses and dysbiosis assessment in IBD cases. In studies where the aim is just to get an overview picture of microbiome, without taxa identification, TGGE seems to be the tool of choice, since it may detect 16SrDNA reads which otherwise in analysis of sequencing data in publicly available database would be collectively grouped as "unidentified" or "unclassified". An additional advantage of TGGE over sequencing is its cost-effectiveness, which becomes particularly evident when the intended analysis focuses on a specific group of bacteria, instead of all species in a microbial community, because the samples can be run in the "mini" version of the TGGE system, which requires a small gel and a lower run time. That was the case of the present study, centered on gram negative, mostly Enterobacteria. A rise in the abundance of E. coli-the principal representative of Enterobacteriaceae-is a marker of dysbiosis in CD patients' gut [10], which apparently, as shown in Figure 3, is linked to the displacement of other unclassified members of this 


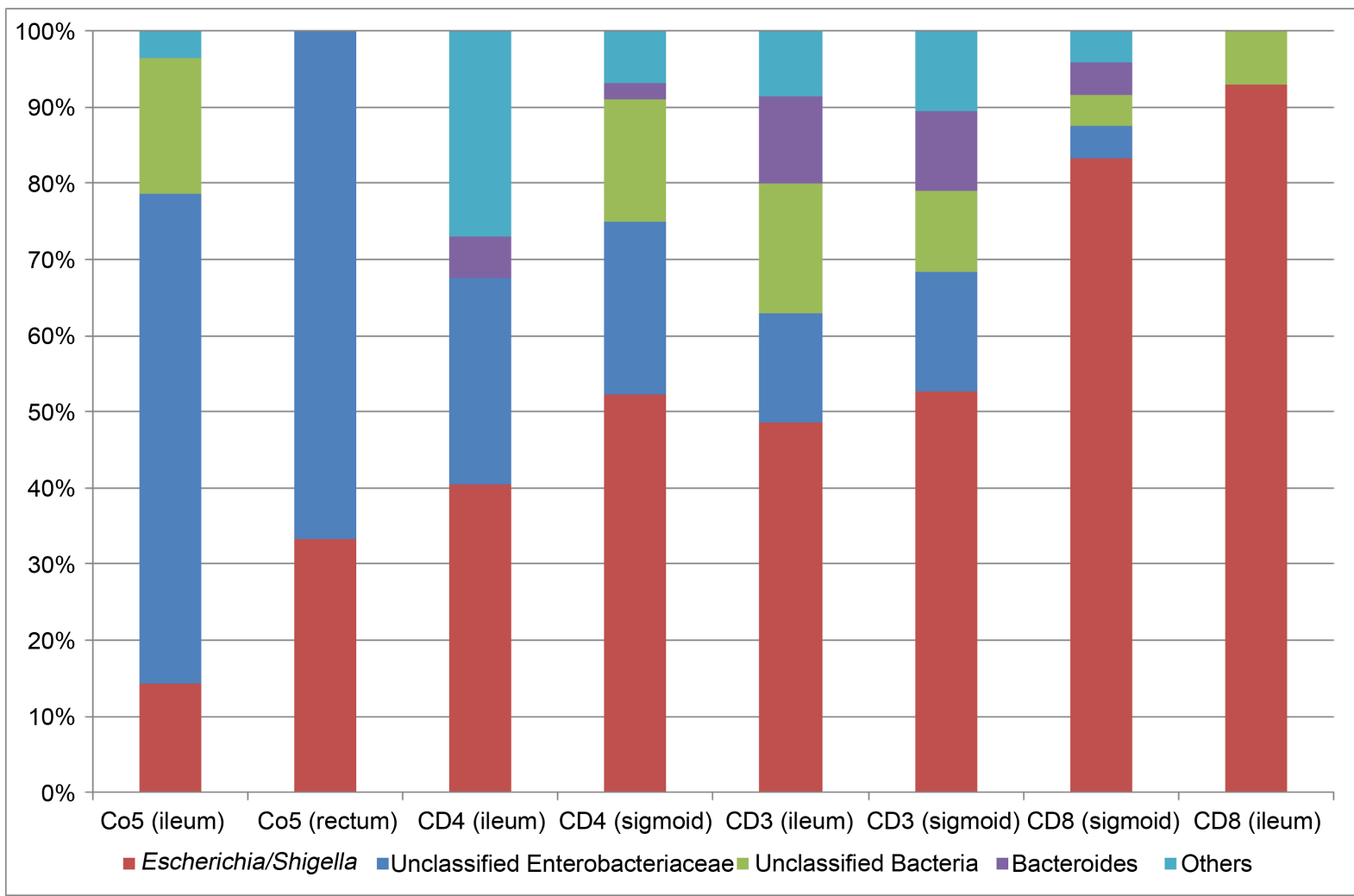

Figure 3. Relative abundance of distinct bacteria taxa in biopsies samples from a control subject (Co5) and 3 CD patients (CD3, CD4, and CD8) as detected by Ion torrent sequencing of 16SV6rDNA amplicons.

family. However, the technique has also its limitations, the most evident of which seem to be occasional lack of data reproducibility due to gel manufacture and silver staining variations. In any case, taking in account all of the above observations, the TGGE based approach for bacterial diversity assessment can be used at minimum as a supporting analytical tool to $16 \mathrm{SrDNA}$ sequencing.

\section{Acknowledgements}

This work was supported by Fapesp Grant 2013/04475-3 given to J.R.

\section{References}

[1] Kruis, W., Fric, P., Pokrotnieks, J., Lukas, M., Fixa, B., Kascak, M., Kamm, M.A., Weismueller, J., Beglinger, C., Stolte, M., Wolff, C. and Schulze, J. (2004) Maintaining Remission of Ulcerative Colitis with the Probiotic Escherichia coli Nissle 1917 Is as Effective as with Standard Mesalazine. Gut, 53, 1617-1623. http://dx.doi.org/10.1136/gut.2003.037747

[2] Borody, T.J., Warren, E.F., Leis, S., Surace, R. and Ashman, O. (2003) Treatment of Ulcerative Colitis Using Fecal Bacteriotherapy. Journal of Clinical Gastroenterology, 37, 42-47. http://dx.doi.org/10.1097/00004836-200307000-00012

[3] Snyder, L., Peters, J.E., Henkin, T.M. and Champness, W. (2013) Molecular Genetics of Bacteria. 4th Edition, American Society of Microbiology, Washington DC. http://www.asmscience.org/content/book/10.1128/9781555817169

[4] Neefs, J.M., Van de Peer, Y., De Rijk, P., Chapelle, S. and De Wachter, R. (1993) Compilation of Small Ribosomal Subunit RNA Structures. Nucleic Acids Research, 21, 3025-3049. http://dx.doi.org/10.1093/nar/21.13.3025

[5] Andersson, A.F., Lindberg, M., Jakobsson, H., Backhed, F., Nyren, P. and Engstrand, L. (2008) Comparative Analysis of Human Gut Microbiota by Barcoded Pyrosequencing. PloS One, 3, e2836. http://dx.doi.org/10.1371/journal.pone.0002836

[6] Cole, J.R., Wang, Q., Fish, J.A., Chai, B., McGarrell, D.M., Sun, Y., Brown, C.T., Porras-Alfaro, A., Kuske, C.R. and Tiedje, J.M. (2014) Ribosomal Database Project: Data and Tools for High Throughput rRNA Analysis. Nucleic Acids 
Research, 42, D633-D642. http://dx.doi.org/10.1093/nar/gkt1244

[7] De Santis, T.Z., Hugenholtz, P., Larsen, N., Rojas, M., Brodie, E.L., Keller, K., Huber, T., Dalevi, D., Hu, P. and Andersen, G.L. (2006) Greengenes, a Chimera-Checked 16S rRNA Gene Database and Workbench Compatible with ARB. Applied and Environmental Microbiology, 72, 5069-5072. http://dx.doi.org/10.1128/AEM.03006-05

[8] de Souza, H.L., de Carvalho, V.R., Romeiro, F.G. and Rodrigues, J. (2012) Mucosa-Associated but Not Luminal Escherichia coli Is Augmented in Crohn’s Disease and Ulcerative Colitis. Gut Pathogens, 4, 21. http://dx.doi.org/10.1186/1757-4749-4-21

[9] Zoetendal, E.G., Akkermans, A.D. and De Vos, W.M. (1998) Temperature Gradient Gel Electrophoresis Analysis of 16S rRNA from Human Fecal Samples Reveals Stable and Host-Specific Communities of Active Bacteria. Applied and Environmental Microbiology, 64, 3854-3859.

[10] Agus, A., Massier, S., Darfeuille-Michaud, A., et al. (2014) Understanding Host-Adherent-Invasive Escherichia coli Interaction in Crohn's Disease: Opening up New Therapeutic Strategies. BioMedResearch International, 2014, 567929. http://dx.doi.org/10.1155/2014/567929

\section{Submit or recommend next manuscript to SCIRP and we will provide best service for you:}

Accepting pre-submission inquiries through Email, Facebook, Linkedin, Twitter, etc A wide selection of journals (inclusive of 9 subjects, more than 200 journals)

Providing a 24-hour high-quality service

User-friendly online submission system

Fair and swift peer-review system

Efficient typesetting and proofreading procedure

Display of the result of downloads and visits, as well as the number of cited articles

Maximum dissemination of your research work

Submit your manuscript at: http://papersubmission.scirp.org/ 